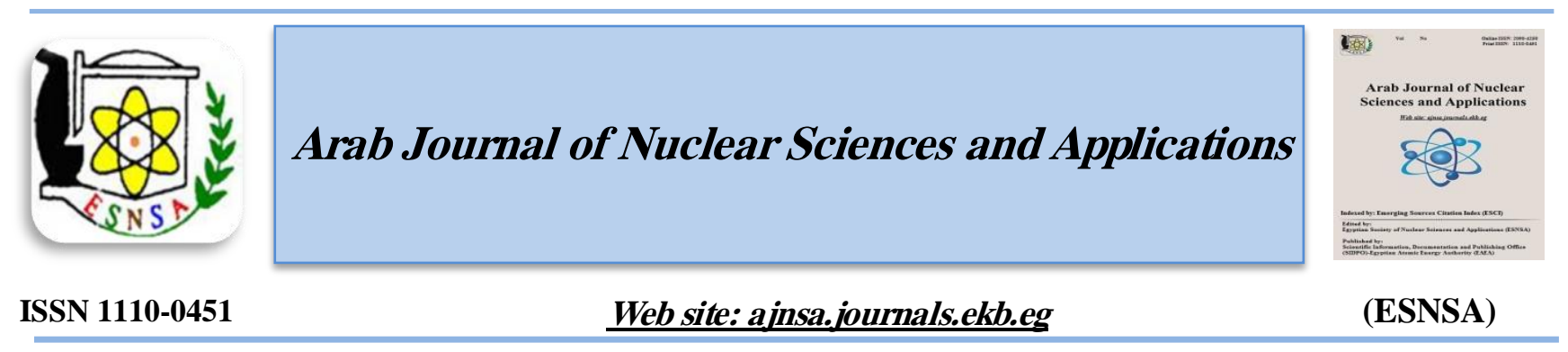

\title{
Landau Damping of Acoustic Waves at the Effect of Variable Dust Size and Charge of Plasma Dust Grains
}

\author{
A. Elbendary \\ Physics Department, Faculty of Science, Tanta University, 31527 Tanta, Egypt
}

Received $5^{\text {th }}$ Oct. 2018 Accepted $8^{\text {th }}$ May 2019

\begin{abstract}
Landau damping is a phenomenon observed in plasma wherein there is an exponential decay in the oscillations of the number density of electrons. Collision less damping of electrical waves in plasma is investigated in the frame of the classical formulation of the problem. In the presence of hybrid nontheremal nonextensive electrons, the effect of variable dust size and charge on the Landau damping of acoustic waves is studied. Using Vlasov - Poisson`s equations with the kinetic theory, Landau damping of dust acoustic waves (DAWs) propagating characteristics with negatively charged dust grains and Maxwellian ion distribution are investigated. The charging of dust grains with different sizes obeys the power law dust size distribution described by the orbital-motion-limited theory. The dependence of the wave frequency $\omega$ on the different dust radii for wave numbers are plotted, also, the Phase diagram of both the grain radius of dusty plasma and the wave number affected the presence of the Landau damping are plotted. There are first, second and third regions in the diagram. This study could be useful for such distribution exists as in the space situations.
\end{abstract}

Keywards: Landau damping/ Acoustic waves/ Effect of variable dust size/ Plasma dust grains

\section{Introduction}

Much more researches focused in Dusty plasma especially in space in the earth`s atmosphere [1-8]. In a study by Barkan et al. [1] dust grains of charges in a plasma gives rise to new one of dust modes. However, the low-frequency of acoustic waves in a magnetized dusty plasma contain electrons, ions and dust particles analysis [2]. Supposing of static and non-static charged dust grains the wave spectra was studied at [3]. Small and finite amplitude electrostatic dust waves in the case of nonlinear propagation were investigated in the low and high density regimes [4]. The study of Dust ionacoustic and dust-acoustic instabilities in dusty plasmas with standard Vlasov approach were previously investigated [5]. The characteristic of dust frequencies was considerably smaller than the corresponding electron or ion quantities [6]. The effects of variable dust size and charge on dust acoustic waves propagating in a hybrid Cairns-Tsallis complex plasma or Nonextensive electron and nonthermal ion with different cases were studied [916].

Because of Landau, damping at dusty plasma with different sizes is similar to some other phenomena in the dusty plasma; much more investigations still remain needed. Therefore, in the present paper, the author studied and investigated the Landau damping taking into consideration the effect of dust size distribution and dust charge. On the other hand, the previous studies in this field mainly focused on dusty plasma, which is of the same sizes and charges of dust grains [17-19]. In fact, the dust grains have many different sizes [20-23], [11]. However, the dust size distribution function is assumed to be a polynomial [24]. At the resonance between particles and waves in plasma, Landau damping is found, which is a collision less damping phenomenon and was first predicted by Landau in 1946 for Langmuir wave [24] , [25]. However, for a Maxwelliian velocity

Corresponding author: atef.elbendary@science.tanta.edu.eg

DOI: 10.21608 /ajnsa.2019.5490.1125

(C) Scientific Information, Documentation and Publishing Office (SIDPO)-EAEA 
distribution, the landau damping of DAWs was studied [26]. Also, Landau damping at either a Lorantzian or nonextensive dusty plasma was investigated [27], [28]. Moreover, the case of the presence of hybrid nonthermal and nonextensive electrons the Landau damping of dust acoustic waves was studied [29]. Due to the data obtained from our survey, in dusty plasma with different sizes of dust grains and charge and with hybrid Carins-Tsallis distributed electrons, the Landau damping of DAWs has not investigated yet. Thus, this study presents, in addition to the above introduction, in section 2, how to obtain the dispersion relation, the linearized Vlasove- poosson`s equations with a kinetic model used for DAWs. While, in section 3, the real and imaginary parts of wave frequency were calculated. Section 4 furnishes a discussion of the characteristics of Landau damping taking into consideration the effect of different sizes and charge distribution of dust grains. In section 5, the conclusion is presented.

\section{Governing equations}

A collision less, nonmagnetized three different components of dusty plasma system which consists of negatively charged massive dust grains of micron size, ions with nonthermal distribution and nonextensive electrons with nonthermal hybrid distribution are considered. Different numbers of $N$ grains of different sizes having radii with the range of $\left[a_{\text {min }}, a_{\text {max }}\right]$ are supposed.

Starting with the one dimension Vlasov - Poisson's equation is given in previous studies [24], [29] for an unmagnetized, collision less dusty plasma.

$\frac{\partial f_{d j}}{\partial t}+v_{d j} \frac{\partial f_{d j}}{\partial x}-\frac{q_{s}}{m_{d j}} \frac{\partial \emptyset}{\partial x} \frac{\partial f_{d j}}{\partial v}=0$

While $f_{d j}$ is represented as the average distribution function of the $j t h$ dust grains (DG) $(j=1,2, \ldots N)$.

The electric field satisfies $E=-\nabla \emptyset$, where

$\nabla^{2} \emptyset=-\left(n_{e}-n_{i}+\sum_{j=1}^{N} \int f_{d j} d^{3} v\right)$

The number densities of ion and electrons are expressed as:

$n_{i}=\mu e^{-s \emptyset}$,

And

$n_{e}=$

$\beta(1+(q-1) s \sigma \emptyset)^{\frac{1}{q-1}+\frac{1}{2}}(1+$

$\left.\frac{16 q \alpha}{3-14 q+15 q^{2}+12 \alpha}\left(-s \sigma \emptyset+(2 q-1) s^{2} \sigma^{2} \emptyset^{2}\right)\right)$,
Where $\beta$ are taken as the normalized number of densities for electrons and $\mu$ for ions. $s=(\mu+$ $\beta \sigma)^{-1}$, and $\sigma=\frac{T_{i}}{T_{e}}$ where $T_{i}$ and $T_{e}$ being the ion and electron temperature respectively.

Equation (2) rewrite as;

$\frac{\partial^{2} \emptyset_{1}}{\partial x^{2}}=-\left(k \emptyset_{1}+\sum_{j=1}^{N} \int_{-\infty}^{\infty} f_{d j} d v\right)$,

$k=s\left(\mu+\frac{\beta(5 q-3) \sigma(-4 \alpha+q(3 q+2)-1)}{24 \alpha+30 q^{2}-28 q+6}\right)$,

In the case of all quantity perturbed and vary with $x, t$ in the form of $\exp i(K x-\omega t)$ Equations (1),(5) are reduced to:

$f_{d j}=\frac{q_{d j}}{m_{d j} \frac{\emptyset_{1}}{\omega}-v} \frac{\partial f_{d j 0}}{\partial v}$,

$\left(K^{2}+k\right) \emptyset_{1}+\sum_{j=1}^{N} \int_{-\infty}^{\infty} f_{d j} d v=0$,

Real and imaginary parts of wave frequency

By equation (7) and (8), the following could be obtained:

$\left(K^{2}+k\right) \emptyset_{1}+\sum_{j=1}^{N} \frac{q_{d j}}{m_{d j}} \int_{-\infty}^{\infty} \frac{\emptyset_{1}}{\frac{\omega}{K}-v} \frac{\partial f_{d j 0}}{\partial v} \partial v=0$,

This gives the dispersion relation of the DAWs, as follows:

$$
\begin{aligned}
& D(\omega, K)=1+\frac{1}{\left(K^{2}+k\right)} \sum_{j=1}^{N} \frac{q_{d j}}{m_{d j}} \int_{-\infty}^{\infty} \frac{\partial v}{\frac{\omega}{K}-v} \frac{\partial f_{d j 0}}{\partial v}=0 \\
& \text { While, } f_{d j 0}=\frac{n_{d j 0}}{\sqrt{2 \pi} v_{t j}} \exp \left(-\frac{v^{2}}{2 v_{t j}^{2}}\right)
\end{aligned}
$$

Where $D(\omega, K)$ at equation (10) is the longitudinal dielectric. The value of $\omega$ is supposed to be complex and could be rewritten as $\omega=\omega_{r}+i \omega_{i}$ where the real part is $\omega_{r}$ and the imaginary part is $\omega_{i}$. By Taylor expansion; equation (10), for dust acoustic waves, $v \ll \frac{\omega}{K^{\prime}}$

$$
\begin{aligned}
& D_{r}\left(\omega_{r}, K\right)=1-\frac{1}{\left(K^{2}+k\right)} \sum_{j=1}^{N} \frac{q_{d j}}{m_{d j}}\left[\frac{n_{d j 0} K^{2}}{\omega_{r}^{2}}(1+\right. \\
& \left.\left.\frac{3 K^{2} v_{t j}^{2}}{\omega_{r}^{2}}\right)\right]=0, \\
& D_{i}\left(\omega_{r}, K\right)= \\
& \sqrt{\frac{\pi}{2}} \frac{1}{\left(K^{2}+k\right)} \frac{\omega_{r}}{K} \sum_{j=1}^{N} \frac{q_{d j}}{m_{d j}}\left[\frac{n_{d j 0}}{v_{t j}^{3}} \exp \left(-\frac{\omega_{r}^{2}}{2 K^{2} v_{t j}^{2}}\right)\right]=0
\end{aligned}
$$


And then by equations (12) and (13) we get,

$$
\begin{gathered}
\omega_{r}^{2}=\frac{K^{2}}{\left(K^{2}+k\right)} \sum_{j=1}^{N} n_{d j 0} \frac{q_{d j}}{m_{d j}}, \\
\omega_{i}^{2}=-\sqrt{\frac{\pi}{8}} \frac{\omega_{r}^{4}}{K^{3}} \sum_{j=1}^{N} \frac{1}{v_{t j}^{3}}\left[\exp \left(-\frac{\omega_{r}^{2}}{2 K^{2} v_{t j}^{2}}\right)\right],
\end{gathered}
$$

The Landau damping under the effect of different size dust grains

In this section, the effects of different sizes distribution on the propagating frequency of dust acoustic waves at equations (12) and (13) are studied. Firstly, the radius $a$ of DG is considered to be satisfying

$\int_{a_{\text {min }}}^{a_{\text {max }}} n(a) d a=N_{\text {tot }}$, where $a_{\text {max }}>a>a_{\text {min }}$, $Z_{d j}=4 \pi \varepsilon_{0} V_{0} a_{j} / e, m_{d j}=\left(\frac{4}{3}\right) \pi \rho_{d} a_{j}^{3}$ and $\rho_{d}$ are the density of dust grains, at equilibrium, $V_{0}$ are the electric surface potential and $\varepsilon_{0}$ are the vacuum permittivity. Replacing the summations by integrals with boundaries $a_{\min }, \quad a_{\max }$ according to ElTaibany et al. [11] where the DG radius $a$ satisfies the following equation:

$\frac{\partial}{\partial \omega_{r}} D_{r}\left(\omega_{r}, K\right) \approx \frac{2 K^{2}}{\left(K^{2}+k\right) \omega_{r}^{3}} \sum_{j=1}^{N} \frac{n_{d j 0} q_{d j}}{m_{d j}}$

Then, the Landau damping of DAWs can be calculated by employing the previously reported formula [27], [28], [29].

$\omega_{i}=-\frac{D_{i}\left(\omega_{r}, K\right)}{\frac{\partial}{\partial \omega_{r}} D_{r}\left(\omega_{r}, K\right)}$

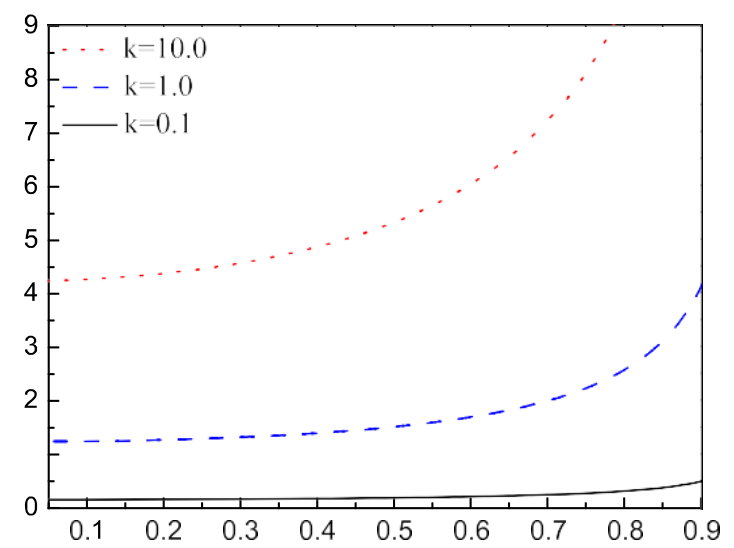

Fig. (1): the wave frequency $\omega$ as the dependence on the difference between dust radius $a(\mu m)$ for_different of reciprocal of wavelength

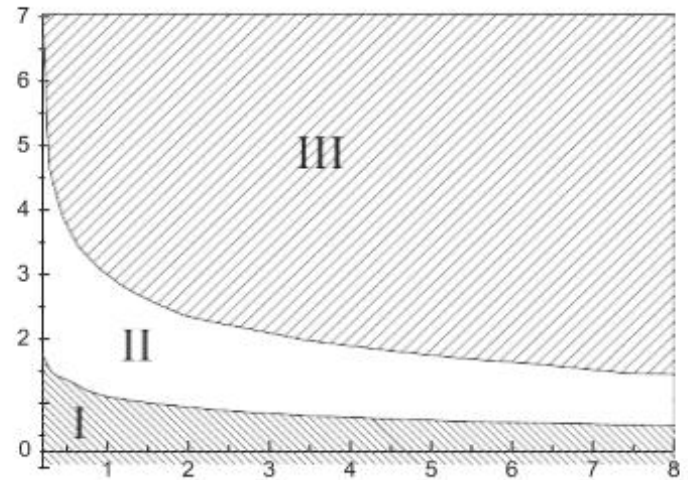

Fig. (2): Landau damping as a function of both the grain radius of DG and the wave number. There are first, second and third regions

In first and third regions I and III, the Landau damping increases while it decreases in the second region. It is easy to observe that the landau damping decreases with increasing the dust grain size radius and increases with increasing the wave number. While it decreases in the case of a wave number greater than 0.1 and less than 1 .

\section{Conclusions}

Landau damping and collision less damping of electrical waves in plasma is investigated in the frame of the classical formulation of the problem taking into consideration the effects of variable dust sizes and charge of dusty grains and under the effect of hybrid Cairns-Tsallis complex plasma. By applying the kinetic theory to Vlasov-Poisson equations, the dispersion relation discussed and obtained for this wave mode. It is found that the nonthermal and the nonextensive parameters have an influence on the wave velocity. Also, the variable dust size distribution has a greater effect on the real part of DAWs frequency and it increases as radius of the dust grain (DG) increases. Landau damping as a function of both the grain radius of DG and the wave number is plotted. There are first, second and third regions. It is found that the frequency of the wave increases as the dust size increases or decreases and as the difference between dust size increases and also as the wave number increases except in the case of wave number greater than 0.1 and less than 1 , it decreases. The result of this study can be useful for understanding Landau damping of DAWs in space and laboratory dust plasma, where the variable dust sizes obey the hybrid nonthermal nonextensive distribution. 


\section{References}

1-Barkan, A., Merlino, R. L., D`angelo, N. (1995) Laboratory observation of the dust acoustic wave mode, Phy. Plasma 2(10), 3563-3565.

2-Rao, N. N. (1993) Low-frequency waves in magnetized dusty plasmas, J. Plasma Phys. 49, 3, 375.

3-Shukla, P.K. (1992) Low-frequency modes in dusty plasmas, Phys. Scr. 45, 5, 504.

4-Rao, N. N., Shulka, P. K. (2001) Nonlinear waves in dense dusty plasmas with high fugacity, Phys. of Plasma 8, 370.

5-Rosenberg, M. (1993) Ion- and dust-acoustic instabilities in dusty plasmas, Plant Space Sci. 41, 229.

6-Verheest, F. (1996) Waves and instabilities in dusty space plasmas, Space Sci. Rev. 77,(3-4), 267.

7-Mamun, A. A. and Shulka, P. K. (2011) Discoveries of waves in dusty plasmas, J. Plasma Phys., vol. 77, no. 4, pp. 437-455.

8-Shulka, P. K. and Mamun, A. A. (2002) Introduction to dust plasma physics, CRC press.

9-Meuris, v. (1997) The influence of a dust size distribution on dust acoustic mode, Plant. Space Sci., vol. 45, no. 9, pp. 1171-1174.

10-Brattli, A., Havnes, O., and melandso, F. (1997) The effect of a dust size distribution on dust acoustic waves, J. Plasma Phys., vol. 58, no. 4, pp. 691-704.

11-EL-Taibany, W. F., EL-Siragy, N. M., Behery, E. E., Elbendary, A. A., and Taha, R. M. (2018) The effects of variable dust size and charge on dust acoustic waves propagating in a hybrid Cairns-Tsallis complex plasma, Indian J.Phys. 92(5), 661-668.

12-Benzekka, M., and Tribeche, M. (2013) Dust acoustic solitons in a charge varying dusty plasma in the presence of ion nonthermality and background nonextensivity, Phys. Plasmas, vol. 20, no. 8, pp. 83702.

13-Silva, R. (1998 ) A Maxwellian Path to the qNonextensive Velocity Distribution Function, phys.lett.A, vol. 249, no. 5-6, pp.401-408.

14-Tsallis, C. (1999) Nonextensive statistics: Theoretical, experimental and computational evidences and connections, Braz. J. Phys., vol.29, no. 1, pp. 1-35.

15-Beck, C. (2001) Dynamical foundation of nonextensive statistical mechanics, Phys. Rev. let., vol. 87, no. 18, pp. 180601.

16-Bacha, M. and Tribeche, M. (2012) Nonextensive dust acoustic waves in a charge varying dusty plasma, Astrophys. Space Sci., vol. 337, no.1, pp. 253-259.
17-Duan, W. S. (2001) Weakly two-dimensional dust acoustic waves, phys. plasmas 8, 3583 .

18-Meuris, P., Verheest, F., Lakhina, G. S. (1997) Influence of dust mass distributions on generalized The Jeans-Buneman Instability in dusty plasmas. Planet. Space sci. 45, 449.

19-19. Duan, S. and Zhao, J. B. (1999) Korteweg-de Vries solitons in inhomogeneous plasma. Phys. Plasmas 6, 3484.

20-Meuris, P. (1997) the influence of a dust size distribution on the dust-acoustic mode Planet. Space sci. $45,1171$.

21-Chen, J. H. and Duan, W. S. (2010) Effects of dust size distribution on instability of obliquely propagating waves in a magnetized dusty plasma phys. plasmas 17, 063701.

22-He, G. J. and Duan, W. S. (2008) Effects of dust size distribution on dust acoustic waves in magnetized two-ion-temperature dusty plasmas, phys. plasmas 15(4), 043702.

23-Lin, M. and Duan, W. S. (2009) The effect of dust size distribution on envelope wave packet in magnetized two-temperature-ion dusty plasma, phys. plasmas 16, 073701.

24-Heng, Z., Yang, Y., Zhang, J., Ren Hong, X. , Lin, M. , Yang, L., Xin, Q., and Duan, W. S. (2014) Landau damping in multi- component dusty plasma, phys. of plasmas 21, 113706.

25-Landau, L.D., (1946) On the vibrations of the electronic plasma, J. Phys. X(1), 25-34.

26-Li, K. M., Kang, L. F., Wang, C.Y., Li, X. and Huang, W.Z.., (2014) Landau damping of dust acoustic waves in dusty plasma, Indian J. Phys. 88(11), 1207.

27-Lee, M. (2007) Landau damping of dust acoustic waves in lorentzian plasma, Phys. plasmas 14(3), 32112.

28-Liu, S. Q., Qiu, H. B., and Li, X. Q.,(2012) Landau damping of dust acoustic waves in the plasma with nonextensive distribution, Physica A 391(23), 5795.

29-El-Taibany, W. F., Zedan, N. A. and Taha, R. M. (2018) Landau damping of dust acoustic waves in the presence of hybrid nonthermal nonextensive electrons, Astrophys Space Sci.363, 129. 